\title{
Optimal control analysis of an SIR epidemic model with constant recruitment
}

\author{
E.A. Bakare ${ }^{1 *}$, A.Nwagwo ${ }^{2}$, E. Danso-Addo ${ }^{3}$ \\ ${ }^{1}$ Department of Mathematics,Federal University Oye Ekiti,Ekiti State, Nigeria \\ ${ }^{2}$ Department of Mathematics, Yaba College of Technology,Lagos, Nigeria \\ ${ }^{3}$ Department of Mathematics, University of Mines and technology, Tarkwa, Ghana \\ *Corresponding author E-mail: emmanuel.bakare@fuoye.edu.ng
}

\begin{abstract}
Copyright (C)2014 Bakare et. al. This is an open access article distributed under the Creative Commons Attribution License Creative Commons Attribution License, which permits unrestricted use, distribution, and reproduction in any medium, provided the original work is properly cited.
\end{abstract}

\begin{abstract}
A mathematical model of an SIR epidemic model with constant recruitment and two control variables using control terms and a deterministic system of differential equation is presented and analyzed mathematically and numerically. We intend to control the susceptible and infected individuals with educational campaign and treatment strategies. We analyzed the model by non-dimensionalizing the system of equations of our SIR epidemic model and derived our basic reproduction number. We aim to minimize the total number of infective individuals and the cost associated with the use of educational campaign and treatment on $[0, T]$. We used Pontryagin's maximum principle to characterize the optimal levels of the two controls. The resulting optimality system is solved numerically. The results show that the optimal combination of treatment and educational campaign strategy required to achieve the set objective will depend on the relative cost of each of the control measures. The results from our simulation is discussed.
\end{abstract}

Keywords: Computational simulations, Disease Free Equilibrium, Optimal control, Pontryagin's Maximum Principle, stability theory.

\section{Introduction}

One of the primary reasons for studying infectious diseases is to improve control and ultimately to eradicate the infection from the population. Models can be a powerful tool in this approach, allowing us to optimize the use of limited resources or simply to target control measures more efficiently. Several forms of control measure exist; all operate by reducing the average amount of transmission between infectious and susceptible individuals. Which control strategy (or mixture of strategies) is used will depend on the disease, the hosts, and the scale of the epidemic[20]. This generation have witnessed a rapid increase in the use of Mathematical models for better understanding of epidemics and disease dynamics. Mathematical models takes into account main factors that govern development of a disease, such as transmission and recovery rates, and predict how the disease will spread over a period of time. Mathematical models have become important tools in analyzing the spread and control of infectious diseases. Some examples on the use of mathematical model for the analyzes of treatment and control of infectious disease can be found in [11, 13, 16, 17, 21, 27, 28], etc. For instance, [13], based on results from the analysis and simulations of their HIV model, suggested universal HIV testing followed by an immediate commencement of antiretroviral therapy for 
those infected as a strategy to drive HIV epidemic towards elimination. Also, [35] proposed an improved Hepatitis $\mathrm{B}$ virus (HBV) model for the treatment of the disease and they claimed that their model control strategy could help reduce death due to HBV remarkably. Traditional epidemiological models divide the whole population into three classes: susceptible, infective and recovered individuals and the spread of an epidemic is governed by the principle of mass action [1], for certain disease, such as, for example, variety of sexually transmitted infectious $[1,6,7,9,30]$. The incidence rate with which individuals becomes infected is normally taken to be bi-linear with respect to susceptible and infected population. The classical transmission S-I-R epidemic was proposed by cooke [8] for epidemics spread in human population via a vector. It is well know that the spread of many infectious diseases can be prevented by vaccination of the susceptible population. furthermore, some infectious provide recovered individuals with short or long treatment against re-infection. This means that it is natural to include the effects of treatment into the mathematical model in order to better represent the actual dynamics of epidemic spread and predict future outbreak. In each case of infectious, the treatment period will vary, as some diseases provide almost life long treatment while others give only a very short-lived non-susceptibility. In most of the research literature, it was frequently assumed that the disease incubation is negligible, in this case, once infected, each susceptible individual becomes infectious instantaneously and later recovers with a temporary acquired immunity or treatment; an epidemic model based on these assumption is called SIR model. An SIR model is essentially a model for an epidemic, it does not provide an epidemiological relevant mechanism for disease epidemiology. The simplest forms of these models are ordinary differential equations (O.D.E) $[14,15]$. In recent years, epidemic models described by ordinary differential equation have been studied and extended by many authors ( see, for examples [5, 12, 18, 25, 26,30,31, 32] and references cited therein). In [23] a system of ODEs with a general incidence term $f(S, I)$ is studied, conditions are found on $f$ under which the standard threshold behaviour occurs; the disease free equilibrium is globally asymptotically stable for $\mathcal{R}_{0}<1$ and endemic equilibrium is globally asymptotically stable for $\mathcal{R}_{0}>1$. In [4], they studied the global stability of an SIR model with vital dynamics first proposed by [14], in their analysis the used the kernel of [5] which is $\gamma$ - distribution.In [31], they studied the stability analysis of an SIR epidemic model with constant recruitment in which the model is formulated under the assumption that all individuals are susceptible; a threshold parameter $\mathcal{R}_{0}$ was obtained to control the disease free and endemic equilibrium.

Optimal control theory is another area of mathematics that is used extensively in controlling the spread of infectious diseases. It is a powerful mathematical tool that can be used to make decisions involving complex biological situation[24]. It is often used in the control of the spread of most diseases for which either vaccine or treatment is available. For example, [11] applied optimal control theory to a set of epidemiological models in their attempt to find the most effective control strategy to minimize the number of individuals who become infected in the course of an epidemic using both treatment and vaccination as control measures. [36] did a related work to the one by [11] but concentrated on an SIR model using only vaccination as their control. Also, the work by [22] used optimal control theory to determine the optimal treatment strategy for the administration of antiretroviral drug (Reverse Transcriptase Inhibitors) in HIV positive individuals. [10] also used optimal control theory to determine the condition for the elimination of tumor cells in an individuals under treatment for Cancer.[34] on the optimal control of vaccination and treatment for an SIR epidemiological model.In their work, they considered an SIR model with variable population size and formulate an optimal control problem to the model with vaccination and treatment as controls. They showed that the disease-free equilibrium is globally asymptotically stable if the basic reproduction number is less than unity while the endemic equilibrium exists and it is globally stable if $R_{0}<1$. They also used the Pontryagin's Maximum Principle to characterize the optimal levels of the two controls. In this work, we included the effect of infected immigrants on the population and also introduced the two control strategies(vaccination and treatments) to reduce the number of Susceptible and infected individuals freely mixing in the population.[19]worked on stability analysis and optimal control of an SIR epidemic model with vaccination. Their work focuses on the study of a nonlinear mathematical SIR epidemic model with a vaccination program. They have discussed the existence and the stability of both the disease free and endemic equilibrium.[2] proposed to study rigorously the optimal control of vaccination and treatments for an SIR Epidemic model with infected immigrants. They included the impact of infected immigrants in order to understand and assess its influence on the transmission dynamics for an SIR Epidemic model. Their main goal is to find the optimal treatment and vaccination strategies in combination that will minimize the cost of vaccination and treatment as well as the number of infectives and to measure the influence of the flow of infected immigrants in the transmission of disease in a population. They perform both qualitative and quantitative analysis of the model with respect to stability of the disease free equilibrium and endemic equilibrium where the two combine controls are in place. They realize that in the absence of infected immigrants the disease free equilibrium exist and is locally asymptotically stable. They established the optimal interventions for the disease control using Pontryagin's Maximum Principle(PMP). Finally, our results on numerical simulations are discussed. 
In this paper, we wish to investigate and analyze, and extended the SIR analysis of [17] and [29] by the inclusion of two control variables to control the susceptible and infected individuals with treatment and educational campaign strategies and the analysis are differs. We derived the dimensionless equations of our SIR epidemic model to reduce the complexity of the problem; a basic reproduction number $\mathcal{R}_{0}$ was also derived and establish to control the equilibria(DFE and EE) while we apply the optimal strategies of disease control by using Pontryagin's Maximum Principle(PMP). The rest of the paper is organized as fellows: the next section, we present the general mathematical frame work, notations, model equations including the basic reproduction number were derived and the SIR model were analyzed. In the third and fourth section, the optimal control technique applied to the model under consideration(SIR Epidemic model) and the numerical simulation results are discussed respectively while the last section deals with a brief conclusion.

\section{Mathematical framework}

The system of equations describing the dynamics of a disease category of the type $S I R$ is derived from the interaction among the compartments: susceptible, infective, and recovered for the model equation.

\subsection{The basic model equations}

The model we presented in this paper is under the frame work of the following non-linear ordinary differential equations.

$$
\begin{aligned}
\frac{d S}{d t} & =A N-\beta S(t)-\lambda I(t) S(t)+\mu R(t) \\
\frac{d I}{d t} & =\lambda I(t) S(t)-(\alpha+\beta+\gamma) I(t) \\
\frac{d R}{d t} & =\gamma I(t)-(\beta+\mu) R(t)
\end{aligned}
$$

The model (1) is related to a model studied in [17] which does not consider vaccination, as well as [15]. We introduce our model through the incorporation of variable population and control strategies into the susceptible and infective class into the classic model in (1). Thus proposed model

$$
\begin{aligned}
\frac{d S}{d t} & =A N-\beta S(t)-\lambda I(t) S(t)+\mu R(t)-u_{2} S(t) \\
\frac{d I}{d t} & =\lambda I(t) S(t)-(\alpha+\beta+\gamma) I(t)-u_{1} I(t) \\
\frac{d R}{d t} & =\gamma I(t)-(\beta+\mu) R(t)+u_{1} I(t)+u_{2} S(t) \\
\frac{d N}{d t} & =A N(t)-\beta N(t)-\alpha I(t)
\end{aligned}
$$

where $N(t)=S(t)+I(t)+R(t)$. We note that the total population size $N$ is a variable. The total population $N$ is stationary for $A=\beta$, declines for $A<\beta$ and grows exponentially for $A>\beta$.

We consider in this work a standard SIR model with bilinear incidence and variable total population. let $\mathrm{S}$ represents the number of susceptible, I represents the number of individuals who are infected, and $\mathrm{R}$ represents the number of individuals who are removed as a result of vaccination or recovery from the diseases with partial immunity or treatment. We note that this model can be applied to a sexually transmitted diseases (such as Gonorrhea).Also, individuals can acquire immunity against the disease either through educational campaign or recovery after treatment for the disease. $\mathrm{A}$ is the recruitment rate, $\beta$ is the natural death, $\lambda$ is the disease transmission rate, $\mu$ is the rate of loss immunity, $u_{1}$ is the fraction of infective that is treated per unit time, $u_{2}$ is the fraction of susceptible that is educational campaign per unit time, $\alpha$ is the disease-induced death rate and $\gamma$ is the natural recovery rate. The total population $N(t)=S(t)+I(t)+R(t)$, it implies that

$$
\frac{d N(t)}{d t}=A N(t)-\beta N-\alpha I
$$

\subsection{Non-dimensionalization of the system}

For our own ease we rewrite these equation in terms of fractions of the individual class by defining $s=\frac{S}{N}, i=\frac{I}{N}$, $r=\frac{R}{N}$ as the proportion for the classes $S, I, R$. For our own convinience we still use $s=S, i=I$ and $r=R$. The system becomes The model equation is now;

$$
\begin{aligned}
\frac{d S}{d t} & =A-\beta S(t)-\lambda I(t) S(t)+\mu R(t)-u_{2} S(t) \\
\frac{d I}{d t} & =\lambda I(t) S(t)-(\alpha+\beta+\gamma) I(t)-u_{1} I(t) \\
\frac{d R}{d t} & =\gamma I(t)-(\beta+\mu) R(t)+u_{1} I(t)+u_{2} S(t)
\end{aligned}
$$


The SIR epidemic model (3) will be analyzed in a biologically feasible region as follows. This region should be feasible for the total populations. If $A$ is a constant then the feasible region for (3) in $\Omega$ is

$\Omega=\left\{(S, I, R) \in \mathbb{R}_{+}^{3}: S+I+R \leq \frac{A}{\beta}\right\}$

Since by (3), $\limsup _{t \rightarrow \infty} S(t) \leq \frac{A}{\beta}$, thus the global attractor of (3) is contain in $\Omega$. Thus the dynamical behaviour of (3) in $\Omega$ and the fate of the disease are determine and control by the basic reproduction number. The basic reproduction number, $\mathcal{R}_{0}$, is the average number of secondary infectious generated when one infected person is introduced into a host population where everyone is susceptible. The basic reproduction number is a threshold quantity thats helps to determine whether an outbreak of infectious disease dies out or spreads in a community. When $\mathcal{R}_{0} \leq 1$, the disease vanishes without the need for any medical strategies. On another case when $\mathcal{R}_{0}>1$, the disease becomes endemic and requires some forms of control strategies to come into place.

Hence,

$\mathcal{R}_{0}=\frac{A \lambda(\beta+\mu)}{\beta\left(\beta+\mu+u_{2}\right)\left(\alpha+\beta+\gamma+u_{1}\right)}$

\subsection{Positivity of solution of model (3)}

Theorem 1: Given $S(0) \geq 0, I(0) \geq 0, R(0) \geq 0$, the solutions $(S(t), I(t), R(t)$ ) of model (3) are positively invariant for all $t>0$. Let $Z_{1}=\sup \{t>0 \mid S>0, I>0, R>0\}$ for the first equation, $\frac{d S}{d t}=A-\beta S-\lambda I S+\mu R-u_{2} S=$ $A+\mu R-\left(\lambda I+\beta+U_{2}\right) S$.

The integrating factor is, $\exp \left(\int_{0}^{t} \lambda I(s) d s+\left(\beta+u_{2}\right) t\right.$. We multiply the inequality above by the integrating factor and we obtain

$\frac{d S(t) \exp \int_{0}^{t} \lambda I(s) d s+\left(\beta+u_{2}\right) t}{d t} \geq(A+\mu R) \exp \left(\int_{0}^{t} \lambda I(s) d s+\left(\beta+u_{2}\right) t\right.$. We solve the inequality and obtain $S(t) \exp \left\{\int_{0}^{t} \lambda I(s) d s+\right.$ $\left.\left(\beta+u_{2}\right) t\right\}-S(0) \geq \int_{0}^{t}(A+\mu R) \exp \left\{\int_{0}^{k} \lambda I(v) d v+\left(\beta+u_{2}\right) k\right\} d k$. Therefore, $S(t)$ becomes

$S(t) \geq S(0) \exp \left\{-\int_{0}^{t} \lambda I(v) d v+\left(\beta+u_{2}\right) t\right\}+\exp \left\{-\int_{0}^{t} \lambda I(v) d v+\left(\beta+u_{2}\right) t\right\} \times \int_{0}^{t}(A+\mu R) \exp \left\{\int_{0}^{k} \lambda I(v) d v+(\beta+\right.$ $\left.\left.u_{2}\right) k\right\} d k>0$. Similarly, it can be shown that $I(t)>0, R(t)>0$ respectively.

\subsection{Boundedness of the solution of model (3)}

Theorem 2: All solutions $(S(t), I(t), R(t))$ of model (3) are Bounded:

Proof: Model(3) only refers to as the total human population from the model equation(3),we obtain

$\frac{d N(t)}{d t}=A-\beta(S+I+R)-\alpha I \leq A-\beta N(t)$. Then $\lim \sup _{t \rightarrow \infty} N(t) \leq \frac{A}{\beta}$.

Therefore all solutions of model (3) are bounded. The feasible region for the total population is $\Omega=\{(S, I, R) \mid$ $\left.S+I+R \leq \frac{A}{\beta}, 0 \leq S \leq S(t), 0 \leq I \leq I(t), 0 \leq R \leq R(t)\right\}$. We defined $\Omega$ as the positively invariant region with respect to the model equation(3), therefore the model equation(3) is mathematically and epidemiologically posed in $\Omega$. Let $\dot{\Omega}$ denote the interior of $\Omega$.

Theorem 3: The region $\Omega \subset \mathcal{R}_{+}^{3}$ is positively invariant for the model (3) with multiple intervention (educational campaign and treatment) with non-negative initial condition in $\mathcal{R}_{+}^{3}$.

\section{Optimal Control Techniques applied to the model under consider- ation}

The control $0 \leq u_{1} \leq 1$ and $0 \leq u_{2} \leq 1$ are the use of treatment and educational campaign. These controls are bounded and our model equation is given below

$$
\begin{aligned}
\frac{d S}{d t} & =A-\beta S(t)-\lambda I(t) S(t)+\mu R(t)-u_{2} S(t) \\
\frac{d I}{d t} & =\lambda I(t) S(t)-(\alpha+\beta+\gamma) I(t)-u_{1} I(t) \\
\frac{d R}{d t} & =\gamma I(t)-(\beta+\mu) R(t)+u_{1} I(t)+u_{2} S(t)
\end{aligned}
$$


with initial conditions

$S(0) \geq 0, I(0) \geq 0, R(0) \geq 0$

The Objective functional is defined as follows

$J\left(u_{1}, u_{2}\right)=\int_{0}^{T}\left(A_{1} I_{h}+A_{2} \frac{u_{1}^{2}}{2}+A_{3} \frac{u_{1}^{2}}{2}\right) d t$

Our goal is to minimize the total number of infective individuals and the cost associated with the use of educational campaign and treatment on $[0, T]$.Now we define the objective functional as

$J\left(u_{1}, u_{2}\right)=\int_{0}^{T}\left(A_{1} I_{h}+A_{2} \frac{u_{1}^{2}}{2}+A_{3} \frac{u_{1}^{2}}{2}\right) d t$

subject to the system of equation (4) with appropriate state initial condition while the Lebesgue measurable control set $U$ is defined as

$U=\left\{\left(u_{1}(t), u_{2}(t) \mid 0 \leq u_{1} \leq 1,0 \leq u_{2} \leq 1, t \in[0, T]\right)\right\}$

where $u_{1}(t)$ and $u_{2}(t)$ are measurable function such that: $0 \leq u_{1} \leq 1,0 \leq u_{2} \leq 1, t \in[0, T] . A_{1}, A_{2}, A_{3}$ is a weight parameter which describes the comparative importance of the two terms in the functional. We consider a quadratic cost on the control, which is the simplest and widest used nonlinear representation of control cost.The quadratic term is particularly chosen to describe the nonlinear behaviour of the cost of implementing the educational campaign and treatments.

\subsection{Formulation of an optimal control problem}

We intend to find $0 \leq u_{1} \leq 1,0 \leq u_{2} \leq 1$ for $t \in[0, T]$, to minimize

$J\left(u_{1}, u_{2}\right)=\int_{0}^{T}\left(A_{1} I_{h}+A_{2} \frac{u_{1}^{2}}{2}+A_{3} \frac{u_{1}^{2}}{2}\right) d t$

subject to the system of equation (4)

$$
\begin{aligned}
& \frac{d S}{d t}=A-\beta S(t)-\lambda I(t) S(t)+\mu R(t)-u_{2} S(t) \\
& \frac{d I}{d t}=\lambda I(t) S(t)-(\alpha+\beta+\gamma) I(t)-u_{1} I(t) \\
& \frac{d R}{d t}=\gamma I(t)-(\beta+\mu) R(t)+u_{1} I(t)+u_{2} S(t)
\end{aligned}
$$

and

$S(0) \geq 0, I(0) \geq 0, R(0) \geq 0$

where $u_{1}(t), u_{2}(t)$ are measurable function such that the control constraint

$$
U=\left\{\left(u_{1}(t), u_{2}(t)\right) \mid 0 \leq u_{1} \leq 1,0 \leq u_{2} \leq 1, t \in[0, T]\right\}
$$

Our goal is to minimize the total number of individuals who are infected while at the same time also minimizing the cost of treatment and the cost of educational campaign of the population given the initial population sizes of all three classes $S(0), I(0)$ and $R(0)$. The first term in the objective functional, $A_{1} I$ stands for the total number of individuals who are infected and is taken as a measure of the death associated with the outbreak. The term $A_{2} \frac{u_{1}^{2}}{2}$ represent the cost of treatment while the term $A_{3} \frac{u_{2}^{2}}{2}$ represent the cost associated with the educational campaign. We consider in this paper a quadratic cost on the control because the cost follows a non-linear representation. Hence $u_{1}, u_{2}$ are Lebesgue integrable . They are piecewise continuous and integrable where $A_{1}, A_{2}$, and $A_{3}$ are relative weights fixed to the cost of minimizing the total number of infected individual, cost of treatment and educational campaign respectively.Hence, we seek an optimal control pair $\left(u_{1}^{*}, u_{2}^{*}\right)$ such that

$J\left(u_{1}^{*}, u_{2}^{*}\right)=\min \left\{J\left(u_{1}, u_{2}\right):\left(u_{1}, u_{2}\right) \in U\right\}$

$U$ is the control set defined by above. 


\subsection{Existence and uniqueness of the control}

Theorem 4.: Suppose the objective functional $J\left(u_{1}, u_{2}\right)=\min \left\{J\left(u_{1}, u_{2}\right)=\int_{0}^{T}\left(A_{1} I_{h}+A_{2} \frac{u_{1}^{2}}{2}+A_{3} \frac{u_{1}^{2}}{2}\right) d t\right.$ where $u=\left\{u_{1}, u_{2}: u_{i}\right.$ measurable $0 \leq u_{1}(t) \leq 1,0 \leq u_{2}(t) \leq 1, t \in\left[t_{0}, T\right] \in \mathbb{R}^{+}$for $\left.i=1,2 \ldots\right\}$ subject to the dynamic constraints of system equations (6) and (9) with $S(0)=S_{0}, I(0)=I_{0}$ and $R(0)=R_{0}$, then there exists an optimal control $u^{*}=\left(u_{1}^{*}, u_{2}^{*}\right)$ such that $\min _{u_{1}, u_{2} \in u} J\left(u_{1}, u_{2}\right)=J\left(u_{1}^{*}, u_{2}^{*}\right)$

Subject to the control system (6) with the initial conditions

Proof.:

To prove the existence of an optimal control pair we use the result in [16] and [Fleming and Rishel (1975)].The control and the state variables are non-negative values and are non-empty. In the minimization problem, the necessary convexity of the objective functional in $u_{1}$ is satisfied. The control variable $u_{1}, u_{2} \in U$ is also convex and closed by definition. The optimal system is bounded which determines compactness needed for the existence of the optimal control. Furthermore, the integrand in the objective functional which is $\left(A_{2} \frac{u_{1}^{2}}{2}+A_{3} \frac{u_{2}^{2}}{2}\right)$ is convex on the control set $U$. There exists constants $b_{1}, b_{2}>0$ and $\beta>1$ such that the integrand of the objective functional $J$ is convex and satisfies $J\left(u_{1}, u_{2}\right) \geq b_{1}\left(\left|\frac{u_{1}^{2}}{2}\right|^{2}+\left|\frac{u_{2}^{2}}{2}\right|^{2}\right)^{\frac{\beta}{2}}-b_{2}$. By standard control arguments involving the bounds on the control, we conclude

$u_{1}^{*}=\left\{\begin{array}{l}0 \text { if } m_{1}^{*} \leq 0, \\ m_{1}^{*} \text { if } 0<m_{1}^{*}<1, \\ 1 \text { if } m_{1}^{*} \geq 1\end{array}\right.$

where

$$
m_{1}^{*}=\frac{\left(\lambda_{I}-\lambda_{R}\right) I}{A_{2}}
$$

$u_{2}^{*}=\left\{\begin{array}{l}0 \text { if } m_{2}^{*} \leq 0, \\ m_{2}^{*} \text { if } 0<m_{2}^{*}<1, \\ 1 \text { if } m_{2}^{*} \geq 1\end{array}\right.$

where

$$
m_{2}^{*}=\frac{\left(\lambda_{S}-\lambda_{R}\right) S}{A_{3}}
$$

By the apriori boundedness of the state system, adjoint system and the resulting Lipschitz structure of the ODEs, we obtain the uniqueness of the optimal control for small T.The uniqueness of the optimal control follows from the uniqueness of the optimality system, which consist of (23), (24) and (25) with characterization (27) and (28). We impose a bound on the length of time interval in order to guarantee the uniqueness of the optimality system. The smallness restriction of the length on the state problem has initial values and the adjoint problem has final values. This restriction is very common in control problems(See 17,24).

\subsection{Necessary conditions of the control}

We use the Pontryagin's Maximum Principle(Pontryagin's et. al; 1962) because it is a constrained control problem. Hence, we give the minimized pointwise Hamiltonian as follows. The necessary conditions that an optimal control must satisfy come from Pontryagin's Maximum Principle(Pontryagin's et. al(1962)). This principle converts the system(1) and equation(3) objective functional into a problem minimizing pointwise a Hamiltonian $H$, with respect to $u_{1}$ and $u_{2}$.

Theorem 5.: Given an optimal control pair $U^{*}(t)=\left(u_{1}^{*}, u_{2}^{*}\right)$ and a solution $X^{*}(t)=\left(S^{*}(t), I^{*}(t), R^{*}(t)\right)$ of the corresponding state system (6) there exist adjoint variables $\lambda_{S}(t), \lambda_{I}(t)$ and $\lambda_{R}(t)$ which satisfies the following: We first derive the Hamiltonian which is given by $H(S, I, R)=\left(A_{1} I_{h}+A_{2} \frac{u_{1}^{2}}{2}+A_{3} \frac{u_{1}^{2}}{2}\right)+\lambda_{S}\left(A-\beta S(t)-\lambda I(t) S(t)+\mu R(t)-u_{2} S(t)\right)+\lambda_{I}(\lambda I(t) S(t)-(\alpha+\beta+\gamma) I(t)-$ 
$\left.u_{1} I(t)\right)+\lambda_{R}\left(\gamma I(t)-(\beta+\mu) R(t)+u_{1} I(t)+u_{2} S(t)\right)$

$$
\begin{gathered}
\lambda_{S}^{\prime}=-\left[\lambda_{S}\left(-\beta-\lambda I-u_{2}\right)+\lambda_{I}(\lambda I)+\lambda_{R}\left(u_{2}\right)\right] \\
=\lambda_{S}\left(\beta+\lambda I+u_{2}\right)-\lambda_{I} \lambda I-\lambda_{R}\left(u_{2}\right) \\
\lambda_{I}^{\prime}=-\left[A_{1}+\lambda_{S}(-\lambda S)+\lambda_{I}\left(\lambda S-\left(\alpha+\beta+\gamma+u_{1}\right)+\lambda_{R}\left(\gamma+u_{1}\right)\right)\right] \\
=-A_{1}+\lambda_{S} \lambda S^{\prime}-\lambda_{I} \lambda S+\lambda_{I}\left(\alpha+\beta+\gamma+u_{1}\right)-\lambda_{R} \gamma-\lambda_{R} u_{1} \\
\lambda_{R}^{\prime}=-\left[\lambda_{S}(-\mu)+\lambda_{R}(-(\beta+\mu))\right] \\
=\mu \lambda_{S}+(\beta+\mu) \lambda_{R}
\end{gathered}
$$

with the final conditions

$\lambda_{S}(T)=\lambda_{I}(T)=\lambda_{R}(T)=0$

Furthermore, we find the optimal control $u_{1}^{*}$ and $u_{2}^{*}$

$u_{1}^{*}=\min \left\{\max \left(0, \frac{\lambda_{I}-\lambda_{R} I}{A_{2}}, 1\right\}\right.$

$u_{2}^{*}=\min \left\{\max \left(0, \frac{\lambda_{S}-\lambda_{R} S}{A_{3}}, 1\right\}\right.$

\section{Proof.}

We form the Hamiltonian $H$ given by

$H(S, I, R)=\left(A_{1} I_{h}+A_{2} \frac{u_{1}^{2}}{2}+A_{3} \frac{u_{1}^{2}}{2}\right)+\frac{u_{1}^{2}}{2}+\lambda_{S}\left(A-\beta S(t)-\lambda I(t) S(t)+\mu R(t)-u_{2} S(t)\right)+\lambda_{I}(\lambda I(t) S(t)-(\alpha+\beta+$ $\left.\gamma) I(t)-u_{1} I(t)\right)+\lambda_{R}\left(\gamma I(t)-(\beta+\mu) R(t)+u_{1} I(t)+u_{2} S(t)\right)$

and the transversality conditions

$\lambda_{S}(T)=\lambda_{I}(T)=\lambda_{R}(T)=0$

Therefore we differentiate the Hamiltonian with respect to $u_{1}$ and $u_{2}$ in the interior of $u_{1}$ and $u_{2}$ we obtain the optimality condition that follows

$$
\begin{aligned}
& \frac{\partial H}{d u_{1}}=A_{2} u_{1}-\lambda_{I} I(t)+\lambda_{R} I(t)=0 \\
& \frac{\partial H}{d u_{2}}=A_{3} u_{2}-\lambda_{S} S(t)+\lambda_{R} S=0
\end{aligned}
$$

From these equations, we obtain the optimal control pair $\left(u_{1}^{*}, u_{2}^{*}\right)$ as stated below

$$
\begin{aligned}
& u_{1}^{*}=\frac{\left(\lambda_{I}-\lambda_{R}\right) I}{A_{2}} \\
& u_{2}^{*}=\frac{\left(\lambda_{S}-\lambda_{R}\right) S}{A_{3}}
\end{aligned}
$$

We impose some bounds on the control variables: $0 \leq u_{1} \leq 1$ and $0 \leq u_{2} \leq 1$ to yield(21) and (22) as required. Therefore our resulting optimality system is given in the next section. 


\subsection{Optimality system}

Therefore, our resulting optimality system is given by:

State equations:

$$
\begin{aligned}
\frac{d S}{d t} & =A-\beta S(t)-\lambda I(t) S(t)+\mu R(t)-u_{2} S(t) \\
\frac{d I}{d t} & =\lambda I(t) S(t)-(\alpha+\beta+\gamma) I(t)-u_{1} I(t) \\
\frac{d R}{d t} & =\gamma I(t)-(\beta+\mu) R(t)+u_{1} I(t)+u_{2} S(t)
\end{aligned}
$$

and

$S(0) \geq 0, I(0) \geq 0, R(0) \geq 0$

Adjoint equations:

$$
\begin{aligned}
& \lambda_{S}^{\prime}=-\frac{\partial H}{\partial S}=-\left[\lambda_{S}\left(-\beta-\lambda I-u_{2}\right)+\lambda_{I}(\lambda I)+\lambda_{R}\left(u_{2}\right)\right] \\
& \lambda_{I}^{\prime}=-\frac{\partial H}{\partial I}=-\left[A_{1}+\lambda_{S}(-\lambda S)+\lambda_{I}\left(\lambda S-\left(\alpha+\beta+\gamma+u_{1}\right)+\lambda_{R}\left(\gamma+u_{1}\right)\right)\right] \\
& \lambda_{R}^{\prime}=-\frac{\partial H}{\partial R}=-\left[\lambda_{S}(-\mu)+\lambda_{R}(-(\beta+\mu))\right]
\end{aligned}
$$

Transversality equations:

$\lambda_{S}(T)=\lambda_{I}(T)=\lambda_{R}(T)=0$.

Characterization of the optimal control $u_{1}^{*}$ and $u_{2}^{*}$ :

$\frac{\partial H}{\partial u_{1}}=\frac{\partial H}{\partial u_{2}}=0$ at $u_{1}=u_{1}^{*}, u_{2}=u_{2}^{*}$ on the set $\left\{t \in[0, T]: 0 \leq u_{1} \leq 1,0 \leq u_{2} \leq 1\right\}$. That is:

$u_{1}^{*}=\left\{\begin{array}{l}0 \text { if } m_{1}^{*} \leq 0, \\ m_{1}^{*} \text { if } 0<m_{1}^{*}<1, \\ 1 \text { if } m_{1}^{*} \geq 1\end{array}\right.$

where $m_{1}^{*}=\frac{\left(\lambda_{I}-\lambda_{R}\right) I}{A_{2}}$

and

$u_{2}^{*}=\left\{\begin{array}{l}0 \text { if } m_{2}^{*} \leq 0, \\ m_{2}^{*} \text { if } 0<m_{2}^{*}<1, \\ 1 \text { if } m_{2}^{*} \geq 1\end{array}\right.$

where $m_{2}^{*}=\frac{\left(\lambda_{S}-\lambda_{R}\right) S}{A_{3}}$

\section{Numerical simulations results and discussions}

Here, we provide some numerical simulations of the epidemiological models which describes the theoretical results and predict the evolution of infectious diseases in the population and we also studied the dynamical behaviour of the models. The model we present here is very general as it can suit any model of diseases like H1N1(Influenza), measles, chicken pox, mumps, etc.

We use the forward-backward sweep method and solved the optimality system numerically. This consists of 6 ordinary differential equations arriving from the state and adjoint equations, coupled with the two controls. In our choices of upper bound for the controls, we supposed that the two controls would not be 100percent effective, so the upper bounds for $u_{1}$ and $u_{2}$ were selected to be between 0 and 1 respectively. The weights in the objective functional are $A_{1}, A_{2}$ and $A_{3}$ respectively. The parameters in Table 1 were choosen based on other literatures and used for our simulations.

We simulate the model in different scenarios.Figure 1 shows scenarios for the state variables of the model for the 
case when the cost differs for the two controls. We observed that the susceptible population with control strategy decreases from the first day of the disease outbreak, while the population of recovered individuals increases from the first day after vaccination. From day 70 the population of susceptible become stable. At day 100 the disease dies out and only susceptible and recovered remains in the population. We also observe that the population of infected individuals with treatment control increases very slowly showing that the treatment control has little effect in reducing the proportion of the infective in the population because the infective grows slowly, the contact rate will be very low and so the diseases will die out in the population. Hence, the proportion of recovered individuals in the system is very large with vaccination control, this implies that there are few infected individuals in the population. This depicts that when educational campaign control was introduced in the population, the proportion of recovered individuals increases. This also shows the effect of educational campaign on the population. Figure 2, represent the three adjoint variables $\lambda_{S}, \lambda_{I}$ and $\lambda_{R}$ in the population and we solve these adjoint by a backward fourth-order Runge-kutta techniques.

Figure 3, represent the optimal control variables $u_{1}$ and $u_{2}$ at a time $t$ play a significant role in minimizing the probability that infected population spread the disease in the host population.

Figure 4(a) depicts scenarios for the infected individuals varying the disease induced death rate. When the disease induced death rate is high the infective population drops gradually and when the disease induced death rate is low the infectious population increases.

Figure 4(b) shows the infected population with varying weight parameter $C_{1}$. We observe that when the weight parameter is high the infected population increases at first and then maintain a steady state and when the weight parameter is low the population of infected individuals grows increasingly.

Figure 4(c) represent the control variables $u_{1}$ with varying weight parameter $\left(C_{3}=2, C_{3}=0\right)$. We observe that when the weight parameter is high the control decreases very fast and when the weight parameter is low the control decreases very slowly.

Figure 5 (a) shows the control variable $u_{2}$ with varying disease transmission rate. This depicts that when the disease transmission rate is high, the control variable $u_{2}$ drops gradually from the upper bound to zero after time $t=0.5$ to its lower bound and when the disease transmission rate is low, the control variable $u_{2}$ drops gradually from the upper bound to zero after time $t=0.65$ to its lower bound.

Figure 5(b). This represent the susceptible population with varying disease induced death rate both decreases very fast at the same rate for the susceptible population. Note that we display the quantity $S, I$ and $R$ as proportions instead of numbers themselves.

The simulation which we carried out were carried out by using the following values: $b=0.02, \alpha=0.1, \mu=0.00137$, $A=0.03, d=0.75, g=0.005, \gamma=0.00137, S(0)=0.55, I(0)=0.05, R(0)=0.35, A_{1}=10, A_{2}=1, A_{3}=2$

$u_{1}^{*}=\frac{\left(\lambda_{I}-\lambda_{R}\right) I}{A_{2}}$

$u_{2}^{*}=\frac{\left(\lambda_{S}-\lambda_{R}\right) S}{A_{3}}$

Considering $0 \leq u_{1} \leq 1,0 \leq u_{2} \leq 1, u_{1}^{*}$ and $u_{2}^{*}$ are given by $u_{1}^{*}=\frac{\left(\lambda_{I}-\lambda_{R}\right) I}{A_{2}}$

$u_{2}^{*}=\frac{\left(\lambda_{S}-\lambda_{R}\right) S}{A_{3}}$

Table 1: Table showing numerical values of parameters used in the simulations.

\begin{tabular}{lccc}
\hline Parameter & Symbol & Value & Source \\
\hline disease induced death rate & $\alpha$ & 0.1 & {$[34]$} \\
disease transmission rate & $d$ & 0.75 & {$[34]$} \\
rate of loss of immunity & $\mu$ & 0.00137 & [estimate] \\
recruitment rate & $A$ & 0.03 & {$[34]$} \\
Natural death rate & $b$ & 0.02 & {$[34]$} \\
natural recovery rate & $g$ & 0.005 & [assumed] \\
\hline
\end{tabular}

\section{Conclusion}

In this paper, we have derived and analyzed a system of deterministic differential equation SIR Epidemic model with constant recruitment. We analyze the model by deriving a non-dimensional system of equation from our original model because it helps to reduce the number of time we might have to solve the equation numerically and also gives us insight into what might be small parameters that could be ignored or treated approximately[Issam Sinjab]. We further verify the positivity and boundedness of the solution of our model where we discovered that our 


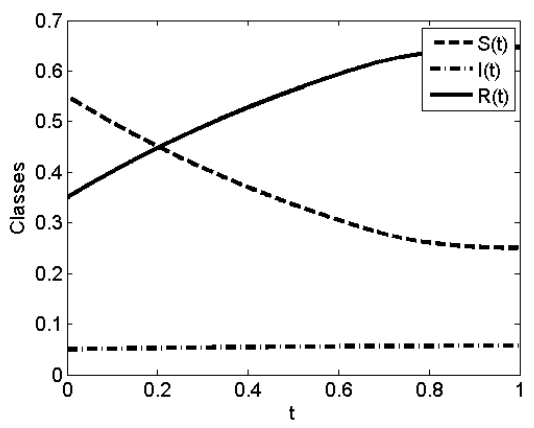

(a)

Figure 1: Simulation showing the Optimal states

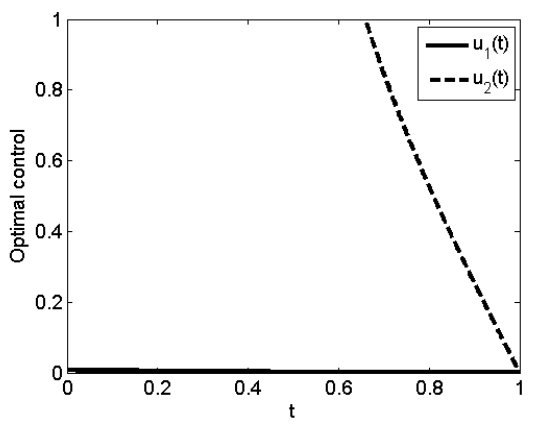

(a)

Figure 2: Simulation showing the Optimal control

model equation is mathematically and epidemiologically well posed in $\omega$. We applied the optimal control theory to our model.In particular, we investigate the stability properties by paying more attention to the basic reproduction number.

We also studied and determined the optimal treatment and educational campaign strategies for our model. We were able to expand on the existing work by putting the missing detail and making reasonable contributions.

We realized that in the course of applying the optimal control, we derived and analyzed the conditions for optimal control of the disease with preventive and treatment measures. From our numerical results, we found that prevention and treatment have a strong impact on the disease control.At this point, we recognized that the objective functional used in the optimization problem can be interpreted or adapted to reflect the actual cost associated with the public health effort in combating any infectious disease.This work can be further extended by introducing stochasticity and partial differential equation(time and space).

\section{Acknowledgements}

The authors thank Prof. F.K Allotey, Director, Institute of Mathematical Sciences, Accra, Ghana for his numerous support. We also wish to thank Prof. Francis Benyah of the Department of Mathematics and Applied Mathematics, University of the Western Cape,Bell-ville, Cape Town, South Africa for his numerous support as well. 


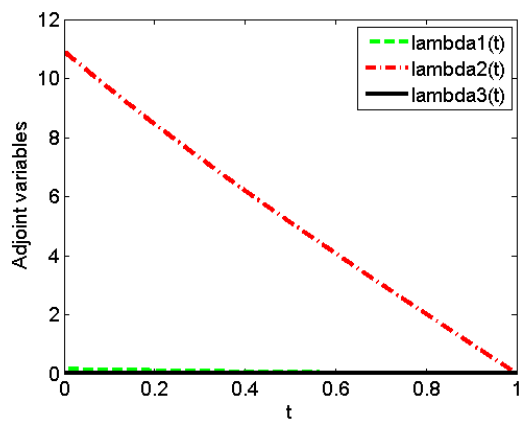

(a)

Figure 3: Simulation showing the Adjoint equations

\section{References}

[1] R.M. Anderson, R.M. May. "Infectious Diseases in Humans Dynamics and Control". Oxford University Press, Oxford,(1991).

[2] E. A Bakare, Yongzheng Sun. "On the Optimal Control of Vaccination and treatments for an SIR-Epidemic with infected immigrants",accepted in Applied Mathematical Sciences, (2014), Hikari Ltd.

[3] E. Beretta, Y.Takeuchi "Convergence results in SIR epidemic model with varying population sizes", Nonlinear Anal., 29,(1997), 1909-1921; .

[4] E. Beretta, Y. Takeuchi "Qualitative properties of Chemostat equations with delayes: Boundedness", local and global asymptotic stability,Diff.Equs.Dynam.SyS., 2,(1944) 19-40.

[5] E. Beretta, V. Capasso, F. Rinaldi. "Global stability results for a generalized Lotka- Voltera system with distribution delays", J. Math.Biol., 26, (1988), 661-688.

[6] F. Brauer, "Model for the spread of universally fatal diseases", J.Math.Biol., 28(4), (1990), 451-462; .

[7] F. Brauer, "Some simple epidemic models", Math. Biosci. Engr., 3(1), (2006) 1-15; .

[8] K.L. Cooke ,"Stability analysis for a vector disease model", Rocky Mount. J.Math., 7, (1979),253-263 .

[9] K.L. Cooke , J.A. Yorke, "Some equations modelling growth processes and gonorhea epidemics", Math. Biosci., 16,(1973), 75-101;

[10] K. Fister, J. Donnelly, "Immunotherapy: An optimal control theory approach". Mathematical Biosciences and Engineering, (2005), 499-510.

[11] H. Gaff, E. Schaefer. "Optimal control applied to vaccination and treatment strategies for various epidemiological models". Mathematical Biosciences and Engineering, (2009), 6: 469-492.

[12] S. Gakkhar, K. Negi "Pulse vaccination in SIRS epidemic model with non-monotonic incidence rate", Chaos Solitions fractals, 35, (2008), 626-638;.

[13] R. Granish, C. Gilks, et al. "Universal voluntary HIV testing with immediate antiretroviral therapy as a strategy for elimination of HIV transmission:a mathematical model". The Lancet, 2008. Doi:10.1016/S0140-6736(08)61697-9.

[14] H.W. Hethcote, "Qualitative analysis of Communicable disease models", Math.Biosci., 28,(1976) 335-356; .

[15] H.W. Hethcote, "The mathematics of infectious diseases", SIAM Review, 42,(2000) 599-653; .

[16] Y. Hsieh, S. Sheu., "The effect of density-dependent treatment and behaviour change on the dynamics of HIV transmission". Journal of Mathematical Biology, (2001), 43: 69-80.

[17] H. Joshi, S. Lenhart, et al., "Modeling the effect of information campaign on the HIV epidemic in Uganda". Mathematical Biosciences and Engineering, (2008), 5: 757-770.

[18] Y. Jin , W. Wang, S. Xiao, "An SIRS model with a non-linear incidence rate" , Chaos Solitions fractals, 34,(2007) 1482-1497. 


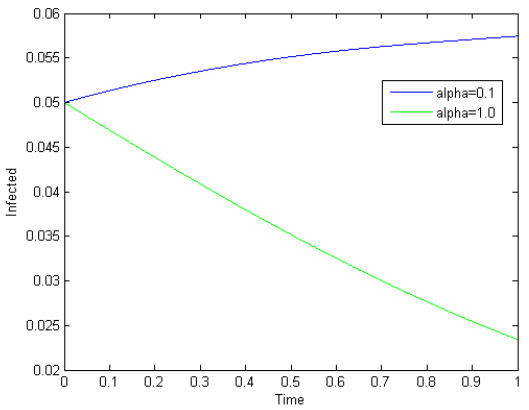

(a)

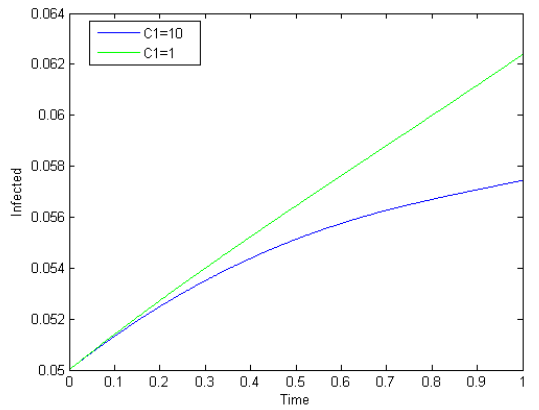

(b)

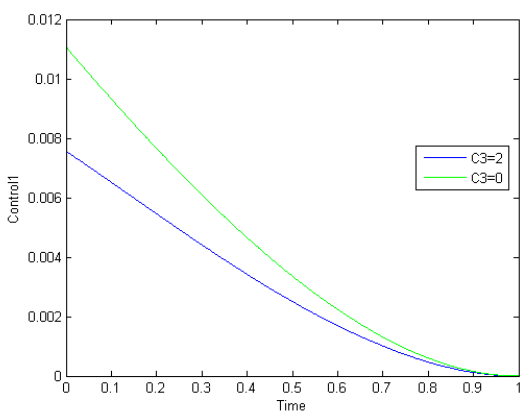

(c)

Figure 4: Simulation showing the infected with varying parameters and $\operatorname{control}\left(u_{1}\right)$ with varying parameter

[19] T.K Kar, Ashim Batabyal., "Stability Analysis and Optimal Control of an SIR Epidemic Model with vaccinations".BioSystems 104 (2011) 127-135.

[20] M. J. Keeling, P. Rohani., "Modelling Infectious Diseases in Humans and Animals". Princeton University Press (2008) $2-3$.

[21] M. Kgosimore, E. Lungu., "The effects of vertical transmission on the spread of HIV/AIDS in the presence of treatment". Mathematical Biosciences and Engineering, (2006), 3: 297-312.

[22] D. Kirschner, S. Lenhart, S. SerBin., "Optimal control of the chemotherapy of HIV". Journal of Mathematical Biology, (1997), 35: 775-792.

[23] A. Korobeinikov, "Global properties of infectious disease models with non-linear incidence", Bull. Math.Biol.,69,(2007) $1871-1886$

[24] S. Lenhart, J. Wortman., "Optimal control applied to biological models". Taylor and Francis, Boca Raton, (2007).

[25] J. Liu , Y. Zhou ," Global stability of an SIRS model with transport-related infection", Chaos Solitions fractals, 40, (2009),145-158; .

[26] J. Mena-Lorca, H.W. Hethcote, "Dynamical models of infections diseases as regular of populations size," J. math.Biol., 30,(1992) 693-716.

[27] Z. Mukandavire, W. Garira., "Sex-structured HIV/AIDS model to analyse the effects of condom use with application to Zimbabwe". Journal Mathematical Biology, (2007), 54: 669-699.

[28] R. Naresh, A. Tripathi, S. Omar., "Modelling the spread of aids epidemic with vertical transmission." Applied Mathematics and Computation, (2006), 178: 262-272.

[29] A. Nwagwo, E.A. Bakare., "Stability Analysis of an SIR Epidemic model and effect of control strategies with constant recruitment", accepted in Applied Mathematical Sciences, (2014), Hikari Ltd.

[30] O.S. Obabiyi A. Nwagwo , "Local asymptotic stability of recurrent diseases", Int.J.Math.Comp., 8(10),(2010) 1-5. 


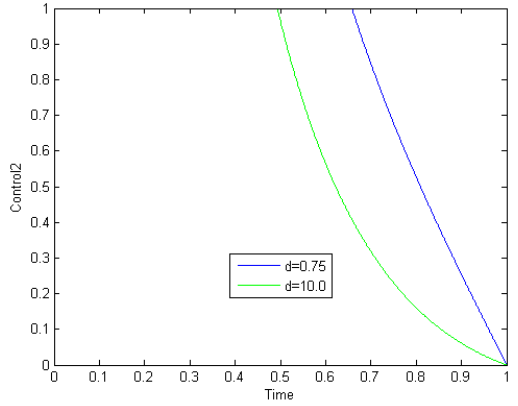

(a)

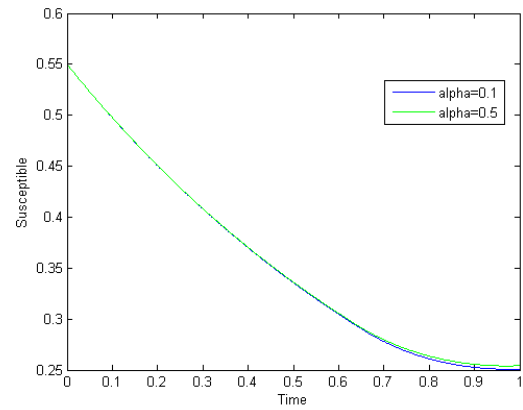

(b)

Figure 5: Simulation showing the susceptible with varying parameter and $\operatorname{control}\left(u_{2}\right)$ with varying parameter

[31] O.S. Obabiyi , A. Nwagwo , I.I.Raji, "Stability analysis of an SIR epidemic model with constant recruitment", FJMS, 42(1), (2010) 21-36.

[32] B. Shulgin, L. Stone, Z. Agur, "Pulse vaccination strategy in the SIR epidemic model", Bull. Math. Biol., 60,(1998) 1123-1148.

[33] Y.Takeuchi Ma W, Beretta E. "Global asymptotic properties of SIR epidemic model with finite incubation time", Nonlinear Anal., 42,(2000), 931-947.

[34] K. Wang, A. Fan, A. Tores., "Global properties of an improved hepatitis B virus model". Nonlinear Analysis: Real World Applications, (2009). Doi:10.1016/j.nonrwa.2009.11.008.

[35] T. Yusuf, F. Benyah., "Optimal Control of Vaccination and Treatment for an SIR epidemiological model". World Journal of Modelling and Simulation. Vol.8(2012) No 3, pp.194-204

[36] G. Zaman, Y. Kang, I. Jung., "Stability analysis and optimal vaccination of an sir epidemic model". BioSystems,(2008), 93: $240-249$. 